Teil I -

Grundlagen: Kulturgeschichtliche, medienpsychologische, -ethische und-rechtliche Zugänge 


\title{
Vom Märchen zur App: Kindheiten im historischen Wandel
}

\section{Regina Ammicht Quinn}

\begin{abstract}
Während die Vorstellung von Kindheit als eigener Lebensphase historisch relativ neu ist, hat der Medienwissenschaftler Neil Postman schon für das Ende des 20. Jahrhunderts das Ende der Kindheit vorhergesagt. Dieses Ende der Kindheit ist für ihn an die Medialisierung von Kindheiten gebunden und ein „disaster of the first order“.

Nun sind Kindheiten heute nicht zu Ende. Aber kontinuierliche Veränderungen machen es nötig, auch einen historischen Blick auf Kinder und Kindheiten zu werfen. Dabei wird deutlich, dass historische Phänomene nicht verschwunden sind, sondern neu gemischt werden und an unterschiedlichen Stellen und in veränderter Form wieder zutage treten. Dieser kulturelle Wandel macht deutlich, dass Kindheiten kein naturgegebenes Phänomen, sondern Zuschreibungen sind - ähnlich wie Zuschreibungen zu Geschlechterrollen oder ethnischen Zugehörigkeiten. Kindheit als Zuschreibung aber bedeutet, dass kontinuierlich nach den Werten dieser Zuschreibung und der Hierarchisierung dieser Werte gefragt werden muss. Diese Hierarchisierungen von Werten dürfen nicht einfach YouTube, Google Classroom oder einer auf Kinder zielende Werbung überlassen werden. Denn an diesen Hierarchisierungen entscheidet sich, ob Kindheiten in gerechter und einer demokratischen Gesellschaft angemessener Weise ermöglicht werden können.
\end{abstract}

\section{Vom Ende der Kindheit}

Kindheiten verändern sich momentan in rasantem Tempo. Manche Kinder und Jugendliche bewohnen Welten, die ihre Großeltern, aber auch ihre Eltern noch nicht kannten - eine Welt der Online Spiele, des kontinuierlichen und unmittelbaren schriftlichen, gesprochenen oder bildlichen Kontakts zu anderen, eine Welt des einfachen Zugangs zu Wissen und Fehlinformationen, zu Phänomenen, die nur im Virtuellen existieren und 
zu virtuellen Phänomenen, die erheblichen Einfluss auf das Selbstbewusstsein, die Entwicklung und das Selbstwertgefühl von Kindern und Jugendlichen haben.

Kindheiten aber haben sich schon immer verändert. Kindheiten waren schon immer plural, unterschiedlich an unterschiedlichen Standorten, in unterschiedlichen sozialen Schichten, Bildungskontexten und politischen Situationen.

Mit Blick auf Mitteleuropa waren in den letzten drei Jahrzehnten vor allem zwei Phänomene die Treiber der rasanten Veränderungen: neue Vorstellungen von Elternschaft und damit auch neue Familienstrukturen, und die Entwicklung hin zu einer digitalisierten Gesellschaft.

Das Wort „Neue Beelterung“ war eines der Kandidaten für das Unwort des Jahres 1997, ein Jahr, nachdem mit „Dolly“ das erste Hausschaf geklont wurde. Beides, der Fortschritt in der Genforschung und der Versuch, eine Sprache für neue soziale Lebensformen zu finden, gehören zusammen. Neue Formen der Elternschaft können biomedizinisch hergestellt werden, und damit wandeln sich die Bilder- und Symbolwelten dessen, was ein Kind ist, welche Rolle und welche Räume ihm zugeschrieben werden. Wenn diese Bilder- und Symbolwelten, aber auch die Räume digitalisiert werden, multiplizieren sie sich. Neue Sichtbarkeiten, Orientierungen und Machtverhältnisse entstehen.

Daher ist es sinnvoll, die aktuellen Fragen von Überwachung und Privatheit von Kindern auch in einen historischen Kontext zu stellen. Es gab Zeiten, in denen Privatheit oder Überwachung keine oder eine andere Bedeutung hatten.

Erik Erikson beschreibt in „Childhood and Society” (1950) die Abhängigkeit individueller Erziehung von historischen Wandlungsprozessen. Kontext und Kultur sind treibende Faktoren für die Konstitution der Lebensphasen, in denen ein Kind den Sinn für das Selbst und die eigene Identität entwickelt. 35 Jahre später nimmt Neil Postman Erikson beim Wort: Kindheit ist geprägt durch die neue mediale Umgebung. Und diese mediale Umgebung führt zum Verschwinden der Kindheit:

„...a new media environment, with television in its center, is leading to a rapid disappearance of childhood in North America. [... C]hildhood will probably not survive the end of this century; and [...] such a state of affairs represents a social disaster of the first order." (Postman 1985: 286)

Postmans Diskussion bezieht sich in den 1980er Jahren insbesondere auf die Rolle des Fernsehens in der Gesellschaft und für die Familien. Was aus heutiger Sicht noch fast nostalgische Vorstellungen weckt, ist für Postman 
Anzeichen einer Kultur, die sich der Technologie unterwirft; Technik sei nicht mehr die Unterstützung des Menschlichen, sondern werde zur kulturprägenden Handlungsmacht (Postman 1992).

Die Vorstellung der Kindheit als eigener Lebensphase ist relativ neu (Winkler 2019). Folgen wir Postman, ist sie aber auch schon wieder zu Ende. Falls das so ist: Welche Kindheit wäre das gewesen? Und gibt es in der Folge neue Kindheiten?

\section{Der Schutz von Kindern: Freiheit und Sicherheit}

Die Art und Weise, wie Kinder geschützt werden, welcher Schutz für sie als notwendig und welche Mittel als angemessen geachtet werden, sind ein Anzeichen dafür, wie Kindheiten wahrgenommen und gestaltet werden.

In manchen Kindheiten mussten Kinder vom Spielen nach Hause kommen, wenn die Straßenlampen angingen. In anderen Kindheiten haben Eltern die Möglichkeit, ihre Kinder mit Hilfe von Tracking-Technologien kontinuierlich zu „begleiten“.

Die Prothelis AG verkauft Tracking Devices für „Hunde, Kinder, Sport und Wertsachen" (www.prothelis.de). Für Kinder ist die "GRETA App“ gedacht, eine Tracking Software mit einem Ortungsgerät, das sich „in der kleinsten Hosentasche“ verstauen lässt. Damit werden „Zäune unsichtbar“ (ebd.), ein Spielgebiet lässt sich individuell festlegen, und die Eltern werden benachrichtigt, wenn das Kind den Bereich verlässt. Die Eltern können auch, so die Website, mit Anrufen „frühzeitig bei unerwarteten Bewegungen des Kindes" gewarnt werden (ebd.).

Was auch immer für erwartbare Bewegungen in die Technologie eingeschrieben sind: Die App macht - repräsentativ - deutlich, dass Kindheiten heute auf vielen Ebenen über das Gewähren von Freiheit und das Fordern von Sicherheit verhandelt werden. Mit der GRETA App soll Freiheit durch Sicherheit hergestellt werden. In den technischen und nicht-technischen Diskursen zu Freiheit und Sicherheit aber wird häufig nicht berücksichtigt, dass eine Güterabwägung zwischen Freiheit und Sicherheit nicht und nie ausreichend ist; hier müssen auch andere Güter, Prinzipien und unterschiedliche Kontexte berücksichtigt werden, für Kindheiten beispielsweise die Fragen nach Sicherheit und Privatheit, Sicherheit und der Herausbildung von Individualität, Sicherheit und Wachstumschancen und anderes.

Die Geschichten, die als Werbung für die GRETA App erzählt werden, unterscheiden sich deutlich von den Geschichten, in denen Gretel (und ihr Bruder Hänsel) eine Rolle spielen. Das Märchen ist eine der ältesten Erzählformen und transportiert damit Bilder, Emotionen und die Fantasie 
früherer Zeitalter mit. Dies geschieht in einem der berühmtesten Märchen Europas in der Besetzung von zwei unschuldigen Kindern, zwei bösen Frauen ((Stief)Mutter und Hexe) und einem schwachen Vater vor dem Hintergrund einer Hungersnot.

Im 19. Jahrhundert hat dieses Märchen Konjunktur:

Die Gebrüder Grimm (Grimm/Grimm 1812-1858) bringen das Märchen in die erste schriftliche Form. In der ersten Fassung 1812 schickt die Mutter die Kinder in den Wald, weil das Essen nicht mehr für alle reicht. Sie treffen auf die Hexe, die Hänsel mästen und essen will, Gretel tötet die Hexe, und die Kinder finden wieder nach Hause, wo die Mutter inzwischen gestorben ist. Die zweite Fassung 1819 malt den Rückweg der Kinder und die Re-Integration in eine Normalität breiter aus. In der Fassung von 1840 ist es nicht mehr die Mutter, die die Kinder los werden will, sondern die Stiefmutter (vgl. dazu Bluhm 2012).

1893 wird Humperdincks Oper „Hänsel und Gretel“ (Libretto: Adelheid Wette) in Weimar uraufgeführt - bis heute eines der am häufigsten gespielten Stücke im Opernrepertoire. In dieser spätromantischen Form (und mit Rücksicht auf das deutlich sensiblere Weimarer Publikum) verändern sich die familiären Verhältnisse und mit ihnen das Verständnis von Kindheit. Zwar sind Mutter und Vater keineswegs Rollenvorbilder - die Mutter jähzornig, der Vater betrunken; beides ist mit einer eindeutigen Klassenzugehörigkeit verbunden. Die Liebe der Eltern zu den Kindern aber wird nicht in Frage gestellt. Die Oper zeigt, wie die Mutter die Kinder zum Beerensuchen in den Wald schickt. Die Kinder finden nicht zurück. Die Eltern suchen überall nach ihnen. Und die Kinder, die nun im dunklen Wald übernachten müssen, singen den Abendsegen, dessen 14 Englein auch dramaturgisch auf der Bühne erscheinen:

Abends will ich schlafen gehn,

Vierzehn Engel um mich stehn:

Zwei zu meinen Häupten, Zwei zu meinen Füßen, Zwei zu meiner Rechten, Zwei zu meiner Linken, Zweie, die mich decken, Zweie, die mich wecken, Zweie, die mich weisen, Zu Himmels-Paradeisen. (2. Akt)

Alleine im Wald zu übernachten scheint für die Geschwister Humperdinck und Wette und das Weimarer Publikum problematisch zu sein - 
darum gibt es schützendes Personal: eine ganze Schutzmauer aus Engeln. Spätestens seit Norbert Elias die Privatisierung und Intimisierung des Schlafes als eines der Kennzeichen des Zivilisationsprozesses beschrieben hat (Elias 1976: 219-230), erscheint diese Situation ziemlich beengt: ein Engel oder auch zwei sehr gerne. Aber vierzehn davon? Diese Überzahl an Schutzpersonal bringt in der Beengtheit eine eigene Freiheit hervor: die Freiheit, sicher im Wald zu schlafen.

Und die Hexe selbst wird am Ende in Lebkuchen verwandelt.

Dieselbe Geschichte - Eltern, zwei Kinder, Not, Gefahr und Überwindung der Gefahr - zeigt in kleinen Verschiebungen der Grundelemente, wie sich innerhalb eines Jahrhunderts die Vorstellung von Kindheit verändert hat: In der 1812 veröffentlichten Version sind die Kinder eine Last, in der nächsten Version wird der Rückweg und damit die Wiederaufnahme in die Familie länger und aufregender. Dann, 1840, ändert sich der Blick auf die Mutterliebe: Keine „richtige“ Mutter würde ihre Kinder in die Gefahr schicken; sie wird also zur Stiefmutter. Und im Ausgang des 19. Jahrhunderts ist dann der Blick auf die Kinder ein anderer: Die Erwachsenen sind zuständig für die Sicherheit der Kinder, religiöse Vorstellungen (und das heißt auch: Vorstellungen von transzendentem, herbeirufbarem Schutz) helfen dabei, und alles wird gut - auch weil es zu Beginn nie so schlecht war. Die GRETA App, die ebenfalls einen Schutzraum für Kinder herstellt, ist ein logischer (über)nächster Schritt.

\section{Kindheitskonzepte im Widerstreit}

Wenn die Geschichte der Kindheit erzählt wird, stößt man auf zwei etablierte, aber widersprüchliche Narrative: Philippe Ariès zeichnet in seiner Geschichte der Kindheit 1960 (D 1975) die Zeit vom 11. bis zum 17. Jahrhundert und den darauffolgenden Wandlungsprozess nach. Ariès, ein enger Freund Foucaults, interessiert sich dafür, wie Mentalitäten rekonstruiert werden können. Bis zum 17. Jahrhundert findet er im Material, das er bearbeitet - vor allem Malerei und Traktate - keine aus der Erwachsenenwelt abgegrenzten und herausgehobenen Kinder. Das gilt auch für das am meisten gezeigte Kind, das Jesuskind, das häufig Gesicht und Gestus eines Erwachsenen hat, auch dort, wo in den Madonnendarstellungen deutlich erotische Motive vorhanden sind. Kinder, so Ariès, waren, sobald sie laufen konnten, in den gemeinsamen Haushalt und dessen Aufgaben integriert. Es gab keine Sonderbereiche des Kindseins: keine spezifische Kleidung, Nahrung oder Unterhaltung. Das gemeinschaftliche Leben zog alle Stände und Altersstufen in einen Sog, mit einem gefühlsmäßig lockeren 
Band zwischen Eltern und Kindern. Die Kinder, beschreibt Ariès, waren frei, ohne vernachlässigt oder verachtet zu werden. Im Übergang zur Neuzeit, in der Renaissance, wird das Kind aus der Gesellschaft der Erwachsenen gerissen. Nun wird strenge Kontrolle zur Grundlage jeder Erziehung, die auf Disziplinierung und Standardisierung des Körpers zielt: „Die Leidenszeit der Kinder beginnt mit der Erfindung der Kindheit.“ (Ariès 1975: 82)

Die Gegengeschichte wird von Psychohistoriker Lloyd deMause erzählt. 1974 erscheint sein Buch „The History of Childhood“, das in Deutschland, provokativ, „Hört ihr die Kinder weinen“ (deMause 1980) heißt. Die Geschichte, die deMause über die Kindheit erzählt, ist ein „Alptraum, aus dem wir gerade erst erwachen. Je weiter wir in der Geschichte der Kindheit zurückgehen, desto unzureichender wird die Pflege der Kinder, die Fürsorge für sie, und desto größer wird die Wahrscheinlichkeit, daß Kinder getötet, ausgesetzt, geschlagen, gequält und sexuell mißbraucht wurden" (deMause 1980: 12). Erst heute gelingt es, so deMause, eine Beziehungsform der Unterstützung zu entwickeln, in der akzeptiert wird, dass das Kind „besser als seine Eltern weiß, was es braucht“ (deMause 1980: 84).

Beide Positionen und Deutungen sind grundlegend kritisiert worden. Es sind zwei unterschiedlich idealisierte Konzepte. Denn es gab mit großer Sicherheit keine Epoche, die Kindern gegenüber völlig gleichgültig war. Der historische Wandel ab dem 17. Jahrhundert war ein diskontinuierlicher Wandel über lange Zeiträume hinweg und voller Brüche; es war ein Wandel, der sich entlang vielfältiger Praktiken von Klasse, Schicht, Geschlecht, Nationalität, regionaler Verankerung und kulturellen Überlieferungen bis heute erstreckt. Während dieser Zeit haben sich spezifische Vorstellungen von Kindheiten herausgebildet, symbolisch gekennzeichnet durch eigene Kleidung, eigene Räume, eigens wahrgenommene Bedürfnisse und die Abtrennung von Sexualität.

Ariès' Einzelanalysen aber liegt eine entscheidende Erkenntnis zugrunde: Kindheit ist kein natürliches, sondern ein historisches Phänomen. Es gibt in allen Gesellschaften Kinder - aber nicht jede Gesellschaft hat eine Vorstellung von Kindheit oder gar ähnliche Vorstellungen von Kindheit (vgl. im Überblick Winkler 2019). Damit ist sein Werk auch politisch zu lesen - als Kritik an der in den 1950er Jahren verbreiteten traditionalistischen These vom Zerfall der Familie (Winkler 2017: 23). Kinder, Kindsein und Kindheit werden in dem langen Übergang hin zur Moderne in neuer Weise in den Blick genommen und reflektiert. Zugleich wird die Geschichte der Kindheit historisch fast ohne Ausnahme als Geschichte der wohlsituierten und weißen Kinder erzählt. 


\section{Das Kind: schuldig/unschuldig; das Kind: öffentlich/privat}

Inmitten dieser historischen Entwicklungen zeigen sich zwei relevante Veränderungen:

Das Kind wird allmählich rein und unschuldig; und dem Kind wird statt der öffentlichen allmählich eine private Rolle zugeschrieben.

Die „Unschuld“ des Kindes ist seit Augustinus ein umkämpftes Terrain. Für ihn ist das Neugeborene von der Erbsünde befleckt, die die Eltern durch Geschlechtsakt und Zeugung an das Kind weitergeben. Die menschliche Natur ist per se sündig (z.B. Augustinus 1888, 1.Buch, 7. Kap.: 5f.). In der mittelalterlichen Vermischung von vorchristlichem und christlichem Dämonenglauben werden dann immer wieder „Wechselbälger“ identifiziert. Wechselbälger werden vom Teufel gezeugt oder von Hexen in der Wiege ausgetauscht - was man manchmal direkt (wenn ein Kind fehlgebildet ist), manchmal erst später merkt (wenn ein Kind kontinuierlich ungehorsam ist). Für Descartes und dessen frühneuzeitlichen Rationalismus wird dann die Sünde zur Sünde der Unvernunft: Alle menschlichen Irrtümer rühren daher, dass ein Mensch Kind gewesen ist. Man(n) muss sich daher von der Kindheit befreien, so wie man sich von einem Übel befreit (Descartes 1955: 253; zit. n. Badinter 1999: 42f.).

Am Übergang von der Aufklärung zur Romantik aber tritt die Unschuld der Kinder in den Vordergrund. Rousseau ist einer der Protagonisten, für den Kinder nicht durch Sünde, sondern durch Unschuld und Reinheit bestimmt sind (als durchgehendes Motiv in Rousseau [1762] 2013). Die Vorstellung von Unschuld und Reinheit des Kindes hat zwei Konsequenzen, die miteinander verwoben sind:

Als erste Konsequenz entwickelt sich eine emotionale Ökonomie in Bezug auf das Kind. In das Kind wird emotional investiert, und damit wird auch eine (vor allem) emotionale Rendite erwartet. Im 19. Jahrhundert wird Mutterliebe zu einem beherrschenden Thema (Badinter 1994), oft verknüpft mit der Opferbereitschaft der Mütter. Bei Vätern findet sich als Zeichen der emotionalen Ökonomie oft eine Sakralisierung des Kindes. "Child is the Father of Man“, so schreibt William Wordsworth (1802; vgl. dazu Winkler 2016). Für ihn ist das (männliche) Kind tief verbunden mit der Natur, die auch als moralischer Richtwert gilt. Das Kind gibt diese Natur-Bindung an den Mann weiter, sodass (zumindest in der englischen Romantik) eine mutterlose Reproduktion des Guten konzipiert wird. Über die englische Romantik hinaus reicht dies bis zu Peter Handke, der 1981 in seiner „Kindergeschichte“ das Kind zum moralischen „Lehrherr[n]“ macht: 
„Er war überzeugt, dass das Kind da ein großes Gesetz verkörperte, welches er selber entweder vergessen oder nie gehabt hatte. War es ihm denn nicht im ersten Monat schon erschienen als sein persönlicher Lehrherr? [...] Das-es-war gab dem Erwachsenen das Wahrheitsmaß an; für ein Leben, wie es sein sollte.“ (Handtke 1981: 63)

Anders als bei Handke, bei dem das bloße Dasein des Kindes genug ist, entwickelt sich in prominenten Teilen der Geistesgeschichte die Vorstellung von Unschuld und Reinheit des Kindes. Aus der Reinheit wird dann die Idee der Formbarkeit des Kindes: Das Kind ist ein unbeschriebenes Blatt und verlangt nach Erziehung. Es ist dann nicht der „Lehrherr“ der Erwachsenen, sondern die Erwachsenen werden zu Lehrherren und das Kind zum Objekt gesellschaftlichen Gestaltungswillens (Winkler 2016, Rose 1999). Parallel dazu, manchmal komplementär, manchmal widersprüchlich, wird die „glückliche Kindheit“ zum „Selbstdarstellungsprojekt des aufstrebenden Bürgertums. Anders als der seine Kinder in ihrer Entwicklung beschränkende Adel, anders auch als das bildungsferne Proletariat, schien nur der Bürger mit seinem Verständnis von Bildung, Arbeit und Moral eine für seine eigenen Kinder und die Kinder einer ganzen Nation richtige Erziehung gewährleisten zu können“ (Winkler 2016; vgl. auch Budde 1994).

Neben dem Wandel hin zur Vorstellung von Unschuld und Reinheit des Kindes ist das Kind eingespannt in das Spannungsfeld von "privat" und „öffentlich“ (Ammicht Quinn 2016: 607f.).

Mit einer unklaren Trennung von Öffentlichkeit und Privatheit sind Kinder vor der Renaissance in gewisser Weise „öffentlich“. In ihnen schreibt sich eine Ahnenreihe fort, sie sind Bausteine des Fortbestands von Familie und Sippe oder der (vorstaatlichen) Nation, auch notwendige Teile der politischen und der ökonomischen Funktion der Familie, die ja Geburtsstätte ständischer Herrschaft und Ort produktiver Arbeit ist. Im Alltag zeigt sich im „ganzen Haus“ auch größte Nähe mit Nicht-Familienangehörigen.

Die allmählich entstehende Spaltung zwischen einem öffentlichen und einem privaten Bereich vor allem ab dem 18. Jahrhundert bringt eine neue Geschlechterordnung und eine neue Generationenordnung hervor, innerhalb derer die Frau zum Privatbereich des Mannes gehört. Kinder gehören zur Frau, mit je nach Geschlecht unterschiedlicher Teilhabe an Öffentlichkeiten des Mannes - bei gleichzeitiger stetiger Institutionalisierung der Bildung. Aufklärerische Freiheit und Gleichheit enden an der Haustürschwelle, wobei im Haus durchaus komplizierte und gegenseitige Abhängigkeitsverhältnisse entstehen können und Männer sich nicht selten in der Rolle eines zusätzlichen Kindes befinden (ebd.). 
Dort, wo Beruf und Familie voneinander getrennt werden, regeln Höflichkeitskonzepte Nähe und Distanz und damit auch das Respektieren der Intimität. Dabei wird - über lange Zeiträume und in unterschiedlichen historischen und kulturellen Bewegungen - das unschuldige Kind zum privaten Kind.

Im 19. Jahrhundert und bis in die Gegenwart führt dies zu einer Idealisierung und gleichzeitigen Entmachtung des Kindes. Das Kind wird niedlich (Winkler 2019: 13). Um niedlich zu sein, muss es aber bestimmten ästhetischen und auch intellektuellen Maßstäben genügen. In den USA wird dann - wie im nationalsozialistischen Deutschland - das als „rassisch anders" wahrgenommene Kind aus der Kindheit herausgeschrieben. Jüdische, schwarze oder andere als „unpassend“ empfundene Kinder sind nicht „niedlich“, sondern (ver)stören die gegenwärtige Gesellschaft und deren wünschbare Zukunft und passen damit nicht in das herrschende Konzept der Kindheit.

\section{Erfolgreiche Kindheiten?}

Ein Verständnis von Kindheit als eigener Lebensphase mit den dazugehörigen eigenen Kinder-Räumen ist heute Konsens. Diese Lebensphase ist zugleich kommerzialisiert und in zunehmendem Maß digitalisiert. Durch die Vervielfältigung der Bilder werden Idealvorstellungen von Kindheit und Kindsein homogenisiert, während zugleich die jeweiligen empirischen Ausgestaltungen plural sind.

Kinder sind „Privatsache“; das wird beispielsweise im Impfstreit und dem damit geforderten Recht der Verfügung der Eltern über den Kinderkörper deutlich. Zugleich werden Kindheiten veröffentlicht - von den Ultraschallbildern Ungeborener über die Abbildung „niedlicher“ Fehler und Peinlichkeiten bis hin zu Lernprogrammen, mit denen, oft unwissend, auch die Lernschwierigkeiten für künftige Profilbildungen relevant werden können.

Die Schutzräume des Privaten waren für Abhängige schon immer ambivalent: Es konnten Räume sein wie Fröbels imaginierter Kinder-Garten, eine Entsprechung des Paradies-Gartens. Fröbel bezieht sich hier auf den Raum einer Zeit der Unschuld, in dem den Kindern die Kindheit geschenkt wird (Fröbel 1848; zit. n. Winkler 2016). Die abgegrenzten Räume aber konnten immer schon zu Räumen der Gewalt werden - im Sinn unmittelbarer, immer wieder auch sexualisierter Gewalt und im Sinn der Gewalt der Marginalisierung, Ausgrenzung, mangelnder Teilhabe oder auch extrem normierter Lebensform und Leistung. In digitalisierten Kinder- 
Welten zeigt sich hier beides: Das Durchbrechen oder die Zerstörung des Schutzraumes erscheint hier als ein Vorgang, der nicht immer bei Ariès idealisierter Freiheit der Kinder unter Erwachsenen endet; das Durchbrechen des Schutzraumes kann problematisch oder bedrohlich sein, etwa dort, wo Informationen, Bilder und Videos nachhaltig verstörend wirken können oder wo die Kontaktaufnahme mit der „realen“ Welt jenseits des geschützten Raums gefährlich sein kann. Gleichzeitig aber kann die Digitalisierung der Kindheit eine große Chance sein: die Chance, die Sichtund Hörbarkeit von Kindern in all ihrer Diversität zu erhöhen.

Der Rückgang der Kinderzahl und der Mortalitätsrate bei Kindern ist eine der Voraussetzungen für die Entstehung einer emotionalen Ökonomie, genauso aber die durch Medialisierung vorangetriebene neue Schätzung des Werts eines Kindes: Kinder sind economically useless, emotionally priceless (Zelizer 1985, Postman 1985). Dies ist zunächst eine menschenfreundliche Entwicklung, die ihre Wurzeln in der Vorstellung der Unschuld des Kindes hat. Die weniger menschenfreundliche Unterseite zeigt sich in Situationen, in denen Kinder für das Lebensglück der Eltern sorgen müssen und damit mit hohen Hypotheken belastet sind, die sie nie zurückzahlen können. Allerdings sind nicht alle Kinder economically useless. Es gibt YouTube-Stars, denen man beim Spielen mit bestellbarem Kinderspielzeug zusehen kann, während sie sehr viel Geld verdienen. Ryan Kaji, 2011 geboren, ist seit 2015 im YouTube-Geschäft mit Videos zum Thema Spielzeug. Für die Jahre 2018 und 2019 gilt er als der bestbezahlte YouTuber, der 22 Millionen US Dollar über die Videos und zusätzliche 26 Millionen US Dollar durch seinen Vertrag mit Walmart verdient. ${ }^{1}$ Man mag Kinder wie ihn niedlich finden, aber sie sind mit einem Mal, wie bereits in der Vormoderne, die ökonomische Versicherung ihrer Eltern.

Das heißt: Historische Einzelphänomene sind nicht verschwunden, sondern werden neu gemischt und treten an unterschiedlichen Stellen und in veränderter Form wieder zutage. Beide Bereiche des historischen Wandels - die Schuld oder Unschuld und die Öffentlichkeit oder Privatheit des Kindes - zeigen sich dort in ihrer Ambivalenz und in einer Ausweitung auf das „System Kindheit“, wo Kindheiten „erfolgreich“ sein müssen.

Noch vor zwei Generationen - in manchen Gegenden und sozialen Schichten viel länger - war die Biografie eines Menschen von Geburt an häufig durch drei Faktoren bestimmt: Genealogie, Geografie, Geschlecht (Ammicht Quinn 2006: 37).

1 https://www.youtube.com/channel/UChGJGhZ9SOOHvBB0Y4DOO_w. 
Die Genealogie bestimmte den Ort in einer Familie, in einem Stand oder einer sozialen Klasse, häufig in einem Beruf oder einer Berufsgruppe, in und auf einem bestimmten Niveau des materiellen und geistigen Lebens: der Sohn des Taglöhners und seiner Frau, des Lehrers und seiner Frau. Die Geografie zeigte die Unterschiede zwischen dem Lehrersohn im Schwarzwalddorf oder in Hamburg. Und das Geschlecht zeichnete jeweils einen von zwei möglichen biographischen Wegen vor: Der Lehrersohn wird wieder Lehrer, die Lehrerstochter heiratet einen.

Bis heute ist für Schul- und Berufserfolg der biografische Einfluss durch den sozialen Status der Familie nicht ausreichend ausgeglichen worden. Dennoch haben diese Faktoren heute ihre absolute Prägekraft verloren. In vieler Hinsicht ist aus Schicksal Wahl geworden. Jede* $\mathrm{r}$ ist ihres und seines Glückes Schmied, und wem das Schmieden nicht gelingt, ist nicht nur unglücklich, sondern an seinem Unglück auch selber schuld. Und genau hier werden Kindheiten zu Folien für Machbarkeitsdenken (Winkler 2019: 12). So böse das klingt, so verständlich ist es. Die Freiheit, die eigene Biografie zu gestalten, wird zu einer Last. Die Last wird besonders schwer, wenn es um Kinder geht. Die glückliche Kindheit als Zielvorstellung des aufstrebenden Bürgertums des 19. Jahrhunderts bekommt dabei die Form der erfolgreichen Kindheit. Kinder (und Eltern) werden an öffentlichen Maßstäben gemessen, und wenn die Maßstäbe nicht erreicht werden, kommt für alle, die mit dem "System Kindheit" befasst sind, die Schuldfrage zurück.

Digitale Technologien sind dann Hilfen für die „richtige“ Entwicklung und das „angemessene“ Lernen; sie demokratisieren den Zugang zu Öffentlichkeiten und das damit verbundene Erfolgsversprechen. Zugleich sind sie Bausteine für die Biografiegestaltung. Dies birgt Chancen und Risiken: Chancen dort, wo hier Kapazitäten der Familien und anderer Beteiligter im „System Kindheit" ergänzt und verstärkt werden können; Risiken dort, wo sich eine neue Möglichkeit des „,childhood engineering“ (Winkler 2016) auftut, die einem Erziehungsziel von Mündigkeit und (auch demokratischer) Freiheit diametral entgegensteht.

\section{Ein Ende der Kindheit?}

Neil Postman war sich sicher, dass das Ende der Kindheit droht, falls die industrialisierten Gesellschaften nicht den Hebel umlegen und die kulturdominierende Wirkung der Medien nicht nur kontrollieren, sondern beenden können. Das ist nicht geschehen. Ein Stück weit sehen wir heute Elemente dieses Endes der Kindheit. Etwa dort, wo die Idee der Gestaltung und Formbarkeit von Kindern und der Gestaltungswille von Erwachsenen 
mit digitaler Hilfe zum „childhood engineering“ wird; dort, wo aufgeweckte Kinder Zugriff auf alles haben, von allen Formen der Sexualität bis zu allen Formen der Gewalt; oder dort, wo der Schonraum als Raum temporärer Entlastung und Freiheit den Kindern geraubt wird, indem durch digitale Analysen individueller Fehler und Talente in Lernprogrammen Kindern eine offene Zukunft verstellt wird.

Zugleich sind die Kindheiten der Kinder heute natürlich nicht zu Ende. Denn es geht nicht darum, dass Kinder heute eine Kindheit haben sollten, die so schön ist wie sie früher auch nicht war. Der nostalgische Anteil in allen kulturpessimistischen Standpunkten benennt häufig einen Teil der Wahrheit - aber eben nur einen Teil.

Die kulturellen Veränderungen zeigen, was Kindheiten sind: kein naturgegebenes Phänomen, sondern eine Zuschreibung. Kindsein und Kindheit sind Zuschreibungen, in denen Vorstellungen von Natur und Kultur eng verwoben sind ähnlich wie in Zuschreibungen von Geschlechterrollen oder ethnischen Zugehörigkeiten. Dies bedeutet, dass solche Zuschreibungen durchaus miteinander konkurrieren können. Vorstellungen von „Kindheit“ können hinter andere Zuschreibungen - wie „Rasse“ oder Klasse - zurücktreten. So werden diese Zuschreibungen auch Aussagen über Strukturen, Machtvorstellungen und Autoritäten einer Gesellschaft: etwa dann, wenn geflüchtete Kinder nicht zuallererst schutzbedürftige Kinder, sondern „Geflüchtete“ sind, oder dort, wo nicht-weiße Kinder zuallererst „Probleme“ sind und nicht Kinder mit einer offenen Zukunft.

Wenn Kindheit eine Zuschreibung ist, dann ist eine Gesellschaft zur Selbstüberprüfung und zum Handeln aufgefordert. Dies gilt auch und gerade dort, wo sich durch digitale Medien, sei es YouTube, Google Classroom oder auf Kinder zielende Werbung bestimmte normative Vorstellungen von "guter" oder „erfolgreicher" Kindheit verfestigen.

Kindheiten sind nicht zu Ende. Sie sind neu und anders mit jeder Generation. Sie sind nicht mehr in einer Weise privat, wie sie es möglicherweise eine relativ kurze Zeit in der Geschichte waren. Kindheiten spielen sich an den vielen Schnittstellen zwischen „öffentlich“ und „privat" ab; es sind Schnittstellen, an denen die Normen der jeweiligen Bereiche immer wieder, manchmal mühsam, ausgehandelt werden. Die Kinderrechtskonvention der Vereinten Nationen ist hier eine notwendige legale Grundlage. Diese Legalität wird mit Hilfe ethischer Überlegungen zu ihrer konkreten Umsetzung je neu ergänzt.

Wenn Kindheiten grundlegend an Gewährung (oder dem Einfordern) von Freiheiten und dem Herstellen (oder dem Infragestellen) von Sicherheiten für Kinder ihre Formen finden, dann ist es eine gesamtgesellschaftliche Aufgabe, Schutzräume als Ermöglichungsräume zur Verfügung zu 
stellen. Es gibt kein allgemeines Rezept dafür, wann, wie und in welchen Mengen und Mischungen Schutz, Fürsorge und Kontrolle in einer digitalisierten Kindheit sinnvoll sind. Dies wird zum einen kleinteilig und individuell, zum anderen politisch und gesellschaftlich ausgehandelt.

Ob Kinder, wie die GRETA App suggeriert, schützenswerte Wertsachen sind, und ob dieser Schutz von imaginierten Engeln oder der Technik kommt: Die Werte, die hier zum Tragen kommen, sind unterschiedlich. Die Fragen, welche Werte essentiell sind, um Kindheiten und das „System Kindheit" zu unterstützen, und wie diese Werte hierarchisiert werden, müssen in den Vordergrund rücken. Denn es muss ständig und ständig neu geklärt werden, wo Sicherheit welchen Freiheiten vorzuziehen ist, ob angestrebter schulischer Erfolg wichtiger ist als Privatheitserwägungen und ob öffentliche Akzeptanz den Vorrang hat vor welchen individuellen Neigungen. Diese Hierarchisierungen von Werten dürfen nicht YouTube, Google Classroom oder einer auf Kinder zielende Werbung überlassen werden. Denn an diesen Hierarchisierungen entscheidet sich, welchen Wert Mündigkeit als Zielvorstellung der gestaltbaren Kindheiten einnimmt und ob Kindheit als Zuschreibung in gerechter und einer demokratischen Gesellschaft angemessener Weise ermöglicht werden kann.

\section{Literatur}

Ammicht Quinn, Regina (2006): Glück - der Ernst des Lebens. Freiburg: Herder.

Ammicht Quinn, Regina (2016): Gender - Aufregung um eine Analysekategorie. In: Stimmen der Zeit 9, S. 600-610.

Ariès, Philippe (1975 [1960]): Geschichte der Kindheit. München: Hanser.

Aurelius Augustinus (1888 u.ö.): Bekenntnisse des heiligen Augustinus. Übersetzung von Otto F. Lachmann. Leipzig: Reclam.

Badinter, Elisabeth (1991): Mutterliebe. Geschichte eines Gefübls vom 17. Jahrhundert bis heute. München: Pieper.

Bluhm, Lothar (2012): Die „Kinder- und Hausmärchen“ der Brüder Grimm. Eine literatur- und kulturwissenschaftliche Einordnung eines, Bestsellers'. Online verfügbar unter: https://iteraturkritik.de/id/17417 (Abfrage am: 22.7.2020).

Budde, Gunilla (1994): Auf dem Weg ins Bürgerleben: Kindheit und Erziehung in deutschen und englischen Bürgerfamilien 1840-1914. Göttingen: Vandehoeck \& Ruprecht. Online verfügbar unter: http://digi20.digitale-sammlungen.de/de/fs1/obj ect/display/bsb00049951_00001.html (Abfrage am: 22.7.2020).

Descartes, René (1955 [1644]): Die Prinzipien der Philosophie. Hamburg: Meiner.

DeMause, Lloyd (1980): Evolution der Kindheit. In: Ders. (Hg.): Hört ihr die Kinder weinen. Eine psychogenetische Geschichte der Kindheit. Frankfurt a. M.: Suhrkamp, S. 12-112 [(1974): The History of Childhood. New York: Harper \& Row]. 
Elias, Norbert (1979 [1939]): Über den Prozess der Zivilisation. Soziogenetische und psychogenetische Untersuchungen. Erster Band: Wandlungen des Verhaltens in den westlichen Oberschichten des Abendlands. Frankfurt a. M.: Suhrkamp.

Erikson, Erik (1950): Childhood and Society. W.W. New York: Norton \& Company.

Fröbel, Friedrich (1848) an Luise Levin in Rendsburg v. 11.11./14.11.1848 (Dresden), Bibliothek für Bildungsgeschichtliche Forschung. Online verfügbar unter: http://bbf.dipf.de/editionen/froebel/fb1848-11-11-01.html (Abfrage am: 22.7.2020).

Grimm, Jacob / Grimm, Wilhelm [1812-1857] (1982): Kinder- und Hausmärchen. Ausgabe letzter Hand. Mit den Originalanmerkungen der Brüder Grimm. Mit einem Anhang sämtlicher, nicht in allen Auflagen veröffentlichter Märchen und Herkunftsnachweisen, hg. von Heinz Rölleke. 3 Bände. Stuttgart: Reclam.

Handtke, Peter (1981): Kindergeschichte. Frankfurt a. M.: Suhrkamp.

Postman, Neil (1985): The Disappearance of Childhood. In: Childhood Education 61 (4), S. 286-293.

Postman, Neil (1992): Technopoly: The Surrender of Culture to Technology. New York: Vintage Books.

Rose, Nikolas S. (1999): Governing the Soul. The Shaping of the Private Self. London: Routledge.

Rousseau, Jean-Jacques (2013 [1762]): Emile oder über die Erziehung. Berlin: Edition Holzinger.

Winkler, Martina (2016): Kindheitsgeschichte, Version: 1.0. In: Docupedia-Zeitgeschichte, 17.10.2016. Online verfügbar unter: http://docupedia.de/zg/Winkler_ki ndheitsgeschichte_v1_de_2016 (Abfrage am: 22.7.2020).

Winkler, Martina (2017): Kindheitsgeschichte. Eine Einführung. Göttingen: Vandenhoek \& Ruprecht.

Winkler, Martina (2019): Kindheit als Konzept aus historischer Perspektive. In: Drerup, Johannes / Schweiger, Gottfried (Hg.): Handbuch Philosophie der Kindheit. Berlin: Metzler, S. 9-17.

Wordworth, William (1802): My Heart Leaps Up. Online verfügbar unter: https://p oets.org/poem/my-heart-leaps (Abfrage am: 22.07.2020).

Zelizer, Viviana A. (1985): Pricing the Priceless Child. The Changing Social Value of Children. New York: Basic Books. 